Acta Crystallographica Section F

Structural Biology and Crystallization Communications

ISSN 1744-3091

Yoshinari Shoyama, ${ }^{a}$ Ayako Takeuchi, ${ }^{a}$ Futoshi Taura, ${ }^{a}$ Taro Tamada, ${ }^{\text {b }}$ Motoyasu Adachi, ${ }^{\text {b }}$ Ryota Kuroki, ${ }^{\text {b }}$ Yukihiro Shoyama ${ }^{a}$ and Satoshi Morimoto $^{\mathrm{a} *}$

${ }^{a}$ Faculty of Pharmaceutical Sciences, Kyushu University, 3-1-1 Maidashi, Higashi-ku, Fukuoka 812-8582, Japan, and ${ }^{\mathbf{b}}$ Neutron Science Research Center, Japan Atomic Energy Research Institute, 2-4 Shirakata-Shirane, Tokai, Ibaraki 319-1195, Japan

Correspondence e-mail:

morimoto@phar.kyushu-u.ac.jp

Received 7 June 2005

Accepted 22 July 2005

Online 30 July 2005

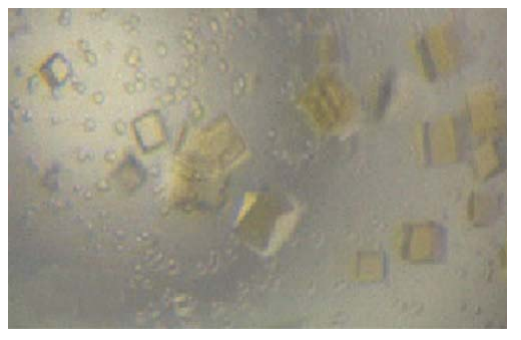

(C) 2005 International Union of Crystallography All rights reserved

\section{Crystallization of $\Delta^{\mathbf{1}}$-tetrahydrocannabinolic acid (THCA) synthase from Cannabis sativa}

$\Delta^{1}$-Tetrahydrocannabinolic acid (THCA) synthase is a novel oxidoreductase that catalyzes the biosynthesis of the psychoactive compound THCA in Cannabis sativa (Mexican strain). In order to investigate the structure-function relationship of THCA synthase, this enzyme was overproduced in insect cells, purified and finally crystallized in $0.1 \mathrm{M}$ HEPES buffer $\mathrm{pH} 7.5$ containing $1.4 \mathrm{M}$ sodium citrate. A single crystal suitable for X-ray diffraction measurement was obtained in $0.09 M$ HEPES buffer $\mathrm{pH} 7.5$ containing $1.26 M$ sodium citrate. The crystal diffracted to $2.7 \AA$ resolution at beamline BL41XU, SPring-8. The crystal belonged to the primitive cubic space group $P 432$, with unit-cell parameters $a=b=c=178.2 \AA$. The calculated Matthews coefficient was approximately 4.1 or $2.0 \AA^{3} \mathrm{Da}^{-1}$ assuming the presence of one or two molecules of THCA synthase in the asymmetric unit, respectively.

\section{Introduction}

Cannabinoids, which are found only in Cannabis sativa, are novel secondary metabolites consisting of alkylresorcinol and monoterpene groups. More than 60 cannabinoids have been isolated from marijuana or fresh Cannabis leaves and their pharmacological properties have been extensively investigated (Turner et al., 1980). Among them, $\Delta^{1}$-tetrahydrocannabinol (THC) is the psychoactive component of marijuana (Gaoni \& Mechoulam, 1964). In addition, this cannabinoid is shown to exert a variety of therapeutic activities such as the relief of nausea caused by cancer chemotherapy (Guzman, 2003) and the suppression of spasticity and pain associated with multiple sclerosis (Baker et al., 2003). Thus, because THC has attracted a great deal of attention, various chemical, pharmacological and biosynthetic studies have been conducted on this cannabinoid (Mechoulam, 1970, 2000).

THC usually accumulates at a quite a low level in the fresh leaves of $C$. sativa and is shown to be derived artificially from the acidic cannabinoid $\Delta^{1}$-tetrahydrocannabinolic acid (THCA) by nonenzymatic decarboxylation during storage and smoking (Yamauchi et al., 1967; Kimura \& Okamoto, 1970). Our biosynthetic studies demonstrated that THCA, which had been believed to be formed<smiles>C=C(C)[C@H]1CCC(C)=CC1c1c(O)cc([13CH3])c(C(=O)O)c1O</smiles>
CBDA

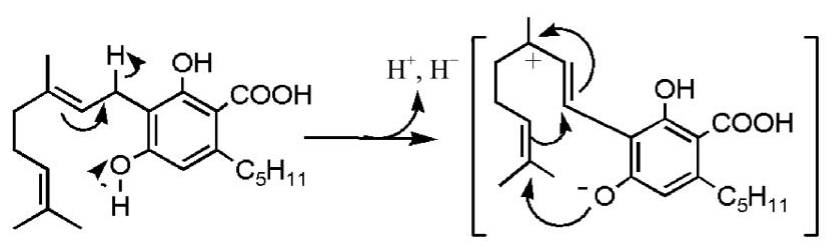
CBGA<smiles>[R]c1c(S)cc2c(c1O)[C@H]1C=C([3H])CC[C@H]1C(C)(O)OC2(C)C</smiles>

THC, $R=\mathrm{H}$ THCA, $R=\mathrm{COOH}$

Figure 1

THCA biosynthesis. THCA synthase catalyzes oxidative cyclization of the monoterpene moiety of CBGA to form THCA. THC is derived from THCA by non-enzymatic decarboxylation. 
through isomerization of cannabidiolic acid (CBDA), was actually derived from cannabigerolic acid (CBGA; Taura et al., 1995, 1996; Fig. 1). Furthermore, we identified and purified an oxidoreductase named THCA synthase which catalyzes THCA biosynthesis in rapidly expanding leaves of $C$. sativa (Taura et al., 1995). Biochemical investigation using the purified enzyme established that THCA synthase oxidatively cyclizes the monoterpene moiety of CBGA to stereospecifically form THCA (Taura et al., 1995; Sirikantaramas et al., 2004). Because enzymes catalyzing such an oxidocyclization have been not identified to date, it is of great interest to precisely reveal the structure of THCA synthase and to understand the mechanism of the THCA synthase reaction.

We have recently cloned the gene encoding THCA synthase and deduced its amino-acid sequence. When we analyzed the features of its primary structure (Sirikantaramas et al., 2004), it became evident that THCA synthase contains a flavin-binding site and has no homology to any proteins for which crystal structures have been determined. Although the site-directed mutagenesis indicated the presence of FAD in THCA synthase to be crucial for oxidocyclization of CBGA, the amino-acid residues involved in stereospecific cyclization of the monoterpene moiety have still not been identified. Therefore, it is impossible to explain the mechanism of the THCA synthase reaction without tertiary structural information.

To obtain more precise knowledge of the structure-function relationship of THCA synthase, we have attempted structural characterization by X-ray crystallography. Here, we describe the crystallization and preliminary X-ray characterization of THCA synthase.

\section{Methods}

\subsection{Expression and purification}

Recombinant THCA synthase (Sirikantaramas et al., 2004) was expressed as a secreted protein in Sf9 insect cells (Invitrogen) using the Bac-to-Bac Baculovirus Expression System (Gibco, BRL). The THCA synthase expression vector was constructed by PCR using a THCA synthase cDNA clone as template (Sirikantaramas et al., 2004). The region coding for 545 residues was amplified using the primers 5'-CGGGATCCATGAATTGCTCAGCATTTT-3' and 5'CTAGCTGAGCTCTTAATGATGATGCGGTG-3'. The resulting fragment was cut with the restriction enzymes BamHI and $S a c \mathrm{I}$ and cloned into pFastBac1 (Gibco BRL). Error-free cDNA clones were identified by sequencing and used to produce recombinant baculovirus in Sf9 cells (ATCC CRL-1711) following the manufacturer's protocol. Sf9 cells were maintained in serum-free SF 900-II medium (Gibco, BRL). For protein production, Sf9 cells were seeded at a cell

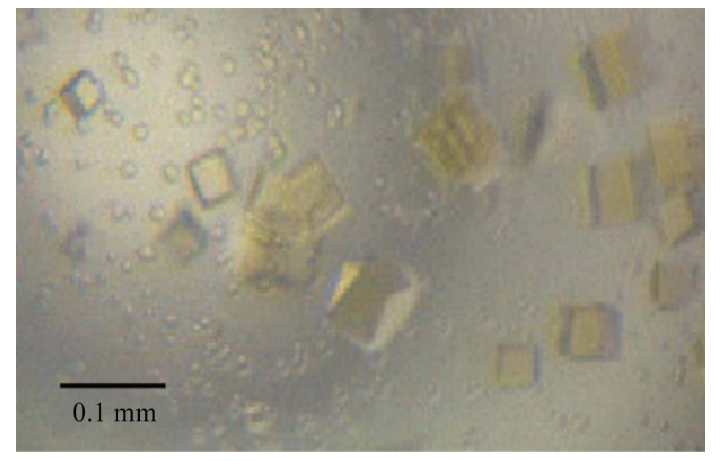

Figure 2

Crystals of THCA synthase. The scale bar is $0.1 \mathrm{~mm}$ in length.
Table 1

Data-collection and reduction statistics.

Values in parentheses are for the highest resolution shell.

\begin{tabular}{ll}
\hline Space group & $P 432$ \\
Unit-cell parameters $(\AA)$ & $a=b=c=178.2$ \\
Solvent content $(\%)$ & $38[2$ molecules per AU] \\
& $69[1$ molecule per AU] \\
Data collection & \\
Wavelength $(\AA)$ & 1.0000 \\
Beamline & BL41XU, SPring8 \\
Measurement range $\left({ }^{\circ}\right)$ & 180 \\
No. of frames & 180 \\
Exposure time per frame $(\mathrm{s})$ & 5 \\
Resolution $(\AA)$ & 2.7 \\
Total reflections & 371920 \\
Unique reflections & 25211 \\
Redundancy & 14.8 \\
$R_{\text {merge }}$ & $0.100(0.306)$ \\
Completeness $(\%)$ & $93.0(88.3)$ \\
\hline
\end{tabular}

density of $1 \times 10^{6}$ cells $\mathrm{ml}^{-1}$ and infected with recombinant virus. The medium was typically harvested on day 3 after infection and THCA synthase was purified from culture supernatant by ion-exchange chromatography. Ion-exchange purification was performed on Toyopearl CM-650 (Tosoh). The supernatant was directly applied onto an ion-exchange column $(3.0 \times 10.0 \mathrm{~cm})$ equilibrated with $50 \mathrm{~m} M$ phosphate buffer $\mathrm{pH}$ 7.0. After washing the column with the same buffer, the protein was eluted with $0.5 \mathrm{M} \mathrm{NaCl}$ in $50 \mathrm{mM}$ phosphate buffer $\mathrm{pH}$ 7.0. Fractions containing THCA synthase activity were collected and dialyzed against $50 \mathrm{~m} M$ phosphate buffer $\mathrm{pH}$ 7.0. The dialysate was applied to the same ion-exchange column $(1.0 \times 15.0 \mathrm{~cm})$. After the column had been washed with $50 \mathrm{mM}$ phosphate buffer $\mathrm{pH} 7.0$, the protein was eluted with a $600 \mathrm{ml}$ linear gradient of $\mathrm{NaCl}(0-0.5 M)$ in $50 \mathrm{~m} M$ phosphate buffer $\mathrm{pH} 7.0$. Eluted protein was further purified on Resource $\mathrm{S}$ (Amersham Biosciences) using a $0-0.5 \mathrm{M}$ linear gradient of $\mathrm{NaCl}$ in $50 \mathrm{mM}$ HEPES $\mathrm{pH} 7.5$ at a flow rate of $1 \mathrm{ml} \mathrm{min}^{-1}$ for $30 \mathrm{~min}$.

\subsection{Crystallization}

Crystallization conditions were screened by the sitting-drop vapour-diffusion method using Crystal Screens I and II (Hampton Research) at $293 \mathrm{~K}$. Drops were prepared by mixing $0.2 \mu \mathrm{l}$ reservoir with $0.2 \mu$ protein solution containing $20 \mathrm{mg} \mathrm{ml}^{-1}$ THCA synthase in $10 \mathrm{~m} M$ HEPES $\mathrm{pH} 7.5$ and were equilibrated by vapour diffusion against reservoir solution. After two weeks, small crystals of THCA synthase were observed in $0.1 M$ HEPES buffer $\mathrm{pH} 7.5$ containing 1.4 $M$ sodium citrate. Finally, a single crystal suitable for X-ray diffraction measurement was obtained using a reservoir solution consisting of $0.09 M$ HEPES $\mathrm{pH} 7.5$ and $1.26 M$ sodium citrate. The size of the crystal was $0.1 \times 0.1 \times 0.1 \mathrm{~mm}$ (Fig. 2 ).

\subsection{X-ray diffraction analysis}

Crystals were harvested in cryoloops and soaked in 0.1 $M$ HEPES buffer pH 7.5 containing $1.4 \mathrm{M}$ sodium citrate and $10 \%$ glycerol. $\mathrm{X}$-ray diffraction data were collected in a cold nitrogen-gas stream at $100 \mathrm{~K}$ using a MAR CCD system and synchrotron radiation $(1.000 \AA$ wavelength) at beamline BL41XU in SPring-8 (Nishiharima, Japan). The oscillation angle was $1.0^{\circ}$ and the exposure time was $5.0 \mathrm{~s}$ per frame. A total of 180 diffraction images were recorded at a camera distance of $170 \mathrm{~mm}$ and were processed using HKL2000 (Otwinowski \& Minor, 1997). 


\section{crystallization communications}

\section{Results and discussion}

THCA synthase overproduced in Sf9 cell showed a molecular weight of $60 \mathrm{kDa}$ on SDS-PAGE, although the theoretical value of the molecular weight calculated from the primary structure of the deduced mature protein was somewhat lower $(58.6 \mathrm{kDa}$ from the primary structure). Since there are at least eight potential N-linked glycosylation sites, the descrepancy in the molecular weight may arise from glycosylation of the THCA synthase. The crystallization conditions for the glycosylated THCA synthase were obtained by the sparse-matrix method and optimized to produce a single crystal suitable for X-ray analysis. Such a crystal with dimensions of $0.1 \times 0.1 \times 0.1 \mathrm{~mm}$ was obtained in $0.09 M$ HEPES buffer $\mathrm{pH} 7.5$ containing $1.26 \mathrm{M}$ sodium citrate. The crystal diffracted to $2.7 \AA$ resolution at beamline BL41XU, SPring-8. Diffraction data processing indicated that the crystal belonged to the primitive cubic space group $P 432$, with unit-cell parameters $a=b=c=178.2 \AA$ (Table 1). The calculated Matthews coefficient was approximately 4.1 or $2.0 \AA^{3} \mathrm{Da}^{-1}$ assuming the presence of one or two molecules of THCA synthase in the asymmetric unit, respectively. The number of observed reflections was 371920 , which were merged into 25211 unique reflections with an $R_{\text {sym }}$ of $0.100\left(R_{\text {sym }}\right.$ in the highest resolution shell was 0.306), a completeness of $93.0 \%$ and multiplicity of around 15 . A search for heavy-atom derivatives for phase determination by multiple isomorphous replacement (MIR) and/or multi-wavelength anomalous dispersion (MAD) is in progress.

We thank Drs K. Maenaka and D. Kohda (Medical Institute of Bioregulation, Kyushu University) for helpful advice on the crystallization of THCA synthase. Thank is also due to M. Kawamoto for data collection at SPring-8.

\section{References}

Baker, D., Pryce, G., Giovannoni, G. \& Thompson, A. J. (2003). Lancet Neurol. 2, 291-298.

Gaoni, R. \& Mechoulam, R. (1964). J. Am. Chem. Soc. 86, 1946-1947.

Guzman, M. (2003). Nature Rev. Cancer, 3, 745-755.

Kimura, M. \& Okamoto, K. (1970). Experientia, 26, 819-820.

Mechoulam, R. (1970). Science, 168, 1159-1166.

Mechoulam, R. (2000). Curr. Pharm. Des. 6, 1313-1322.

Otwinowski, Z. \& Minor, W. (1997). Methods Enzymol. 276, 307-326.

Sirikantaramas, S., Morimoto, S., Shoyama, Y., Ishikawa, Y., Wada, Y., Shoyama, Y. \& Taura, F. (2004). J. Biol. Chem. 279, 39767-39774.

Taura, F., Morimoto, S. \& Shoyama, Y. (1996). J. Biol. Chem. 271, 1741117416.

Taura, F., Morimoto, S., Shoyama, Y. \& Mechoulam, R. (1995). J. Am. Chem. Soc. 117, 9766-9767.

Turner, C. E., Elsohly, M. A. \& Boeren, E. G. (1980). J. Nat. Prod. 43, 169-234.

Yamauchi, T., Shoyama, Y., Aramaki, H., Azuma, T. \& Nishioka, I. (1967). Chem. Pharm. Bull. 15, 1075-1076. 